\title{
Hyperons polarization in heavy-ion collisions
}

\author{
Mircea Baznat ${ }^{1,2, \star}$, Konstantin Gudima ${ }^{1, \star \star}$, Alexander Sorin ${ }^{2,3,4, \star \star \star}$, and Oleg Teryaev ${ }^{2,3,4, \star \star \star \star}$ \\ ${ }^{1}$ Institute of Applied Physics, Academy of Sciences of Moldova, MD-2028 Kishinev, Moldova \\ ${ }^{2}$ Joint Institute for Nuclear Research, 141980 Dubna, Russia \\ ${ }^{3}$ National Research Nuclear University MEPhI (Moscow Engineering Physics Institute), Kashirskoe Shosse \\ 31, 115409 Moscow, Russia \\ ${ }^{4}$ Dubna International University, 141980, Dubna, Russia
}

\begin{abstract}
We study the structure of vorticity and hydrodynamic helicity fields in peripheral heavy-ion collisions using the kinetic Quark-Gluon Strings Model. The angular momentum which is a source of P-odd observables is preserved within this model with a good accuracy. We observe the formation of specific toroidal structures of vorticity field. Their existence is mirrored in the polarization of hyperons of the percent order. The observed qualitative energy dependence of polarization was predicted earlier and is quantified now.
\end{abstract}

\section{Introduction}

The local violation [1] of discrete symmetries in strongly interacting QCD matter is now under intensive theoretical and experimental investigations. The renowned Chiral Magnetic Effect (CME) uses the (C)P-violating (electro)magnetic field emerging in heavy ion collisions in order to probe the (C)P-odd effects in QCD matter.

There is an interesting counterpart of this effect, Chiral Vortical Effect (CVE)[2] due to coupling to P-odd medium vorticity leading to the induced electromagnetic and all conserved-charge currents [3], in particular the baryonic one.

Another important P-odd observable is the baryon polarizatiion. The mechanism analogous to CVE (known as axial vortical effect, see [4] and references therein) leads to induced axial current of strange quarks which may be converted to polarization of $\Lambda$-hyperons $[3,5,6]$. Another mechanism of this polarization is provided by so-called thermal vorticity in the hydrodynamical approach [7].

The zeroth component of axial current and correspondent axial charge are related to hydrodynamical helicity

$$
H \equiv \int d V(\vec{v} \cdot \vec{w})
$$

\footnotetext{
${ }^{\star}$ e-mail: baznat@theor.jinr.ru

$\star \star$ e-mail: gudima@cc.acad.md

$\star \star \star$ e-mail: sorin@theor.jinr.ru

$\star \star \star \star$ e-mail: teryaev@theor.jinr.ru
} 
being the projection of velocity $\vec{v}$ to vorticity $\vec{w}=\operatorname{curl} \vec{v}$. This quantity is manifesting the recently discovered [5] and confirmed [8] phenomenon of the separation, i.e. its mirror behavior with the same magnitudes but different signs in the half-spaces separated by the reaction plane.

The noncentral heavy ion collisions could naturally generate a rotation (global or local, both related to vorticity) with an angular velocity normal to the reaction plane, which is their generic qualitative feature. It is natural to expect that angular momentum conservation plays an essential role in the defining the quantitative properties of vortical effects. At the same time, it remains to be studied to which extent the particles carrying the main part of angular momentum participate in the collisions.

In the current paper we address this problem by performing the extensive numerical simulations. We explore the distribution of angular momentum and find that the role of participant nucleons is relatively small, albeit noticeable. We study in some detail the structure of vorticity field and compare different approaches to polarization calculation. We observed the peculiar toroidal "tire-like" structure manifesting themselves in the polarization of hyperons. We will explore different approaches to polarization calculation which will lead to qualitatively similar results.

\section{Angular momentum conservation in the kinetic model}

The natural source of the P-odd observables in heavy-ion collisions is the pseudovector of angular momentum. The question immediately emerges whether it is conserved in the course of evolution governed by Quark-Gluon String Model (QGSM)[9, 10]. To check this we calculated the angular momentum at various time moments of collision taking into account both the contributions of participants and spectators. We consider the $A u+A u$ collisions with $b=8 \mathrm{fm}$ at $\sqrt{\mathrm{s}}=5 \mathrm{GeV} / u$ typical for future NICA collider. We observed (see figure 1) that the participants carry about $20 \%$ of angular momentum and that the total angular momentum of participants and spectators is conserved with a rather good accuracy.

One may conclude that the angular momentum is under the good control in QGSM model.

We also studied correlations of angular momentum and the hydrodynamic helicity and observed that these quantities vary in accordance with each other. In order to perform this comparison and further calculations, velocity, vorticity and helicity were determined following the earlier suggested [5] procedure when the respective quantities were properly averaged over events and particles within the three-dimensional cells providing the transition from the kinetic to hydrodynamic description.

Let us pass to the corresponding results.

\section{Large-scale structures of vorticity fields}

We start our studies with the qualitative structure of velocity and vorticity fields.

The general structure of velocity field follows the "little bang" pattern which may be quantified by the velocity dependence allowing to extract the "little Hubble" constant. We calculated the dependence of average cell velocity on the transverse distance $\rho=\sqrt{x^{2}+y^{2}}$ and found (see figure 2) that it is consistent with the linear "Hubble" law

$$
<v / c>=v_{0} / c+H \rho .
$$

The Hubble constant $H$ is measured in the units $10^{-22} \mathrm{sec}=30 \mathrm{fm} / \mathrm{c}$ and changes in the range

$$
H=0.024 \div 0.028(\mathrm{fm} / \mathrm{c})^{-1}
$$

It corresponds to the "little Universe" lifetime of about $40 \mathrm{fm} / \mathrm{c}$ which is only twice larger than the collision time. 


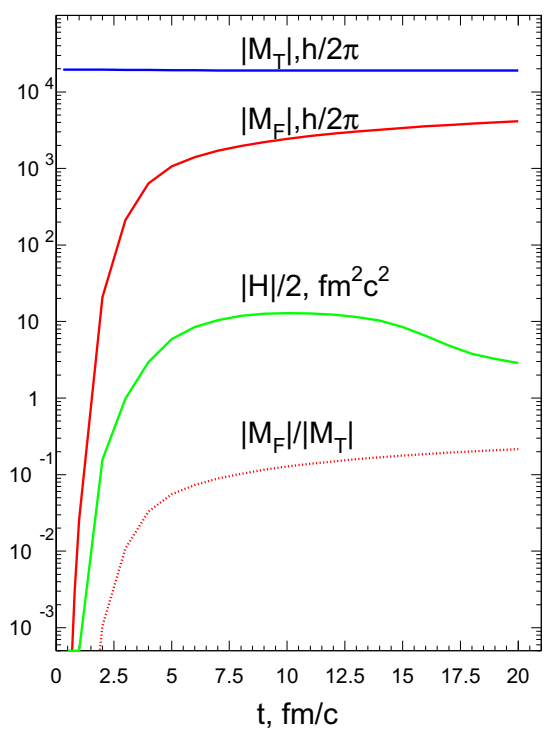

Figure 1. The time dependence of the total $\left(M_{T}\right.$ and fireball $\left(M_{F}\right)$ angular momenta in Planck constant units and that of hydrodynamical helicity.

Our key observation is that while velocity field represent the "little bang" picture, vorticity field form the relatively thin toroidal "tire-like" structures (figure 3), which emerge in the layer (where velocity field changes rapidly) separating the "core" and "corona" regions $[11,12]$ and form the sort of vortex sheet [13].

An interesting property of these structures is that, while emerging due to angular momentum pseudovector $\vec{L}$ in the non-central collisions they do not "remember" the production plane and possess the cylindrical symmetry w.r.t. collisions axis $z$. This may be observed (figure 4) by considering the vortex sheet in the case of the particular direction of $\vec{L}$ along the $y$ axis.

Such behaviour may resemble cyclones appearing at femtoscopic scale.

Let us now discuss the observable signatures of these nice structures.

\section{Hyperon polarization}

We consider hyperon polarization as the observable related to vorticity and helicity [3]. We shall concentrate mostly on $\Lambda$ hyperon production, which has some advantages: they are produced in large numbers, their polarization may be easily determined in their weak decays, and their spin is carried by strange quark.

We compare the two rather distinct methods of determining the hyperon polarization. The first corresponds to its earlier suggested [3] and explored [5] relation to the induced axial current while the second one follows the procedure based on the thermal vorticity [7]. 


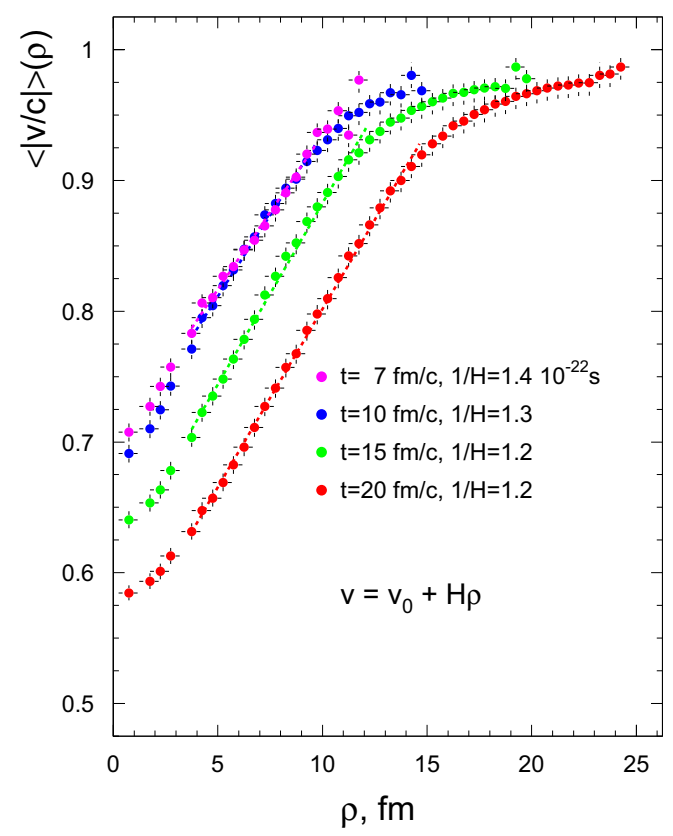

Figure 2. The cell velocity dependence on the transverse distance.

The first method is based on the calculation of strange axial charge

$$
\begin{array}{r}
Q_{5}^{s}=\frac{N_{c}}{2 \pi^{2}} \int d^{3} x \mu^{2}(x) \gamma^{2} \epsilon^{i j k} u_{i} \partial_{j} u_{k} \\
=\frac{<\mu^{2} \gamma^{2}>N_{c} H}{2 \pi^{2}} .
\end{array}
$$

In [5] we used the latter equality exploring the mean-value theorem, while here the spatial variation of strange chemical potential $\mu$ is taken into account. To do so, the description of kinetic distribution functions by the correspondent equilibrium equation was performed, providing the matching of kinetic and thermodynamical descriptions. The average polarization can be estimated by dividing $Q_{5}^{s}$ (2) by the number of $\Lambda$ 's, assuming that the pseudovector of axial current is proportional to the pseudovector of polarization, $Q_{5}^{s} \sim<\Pi_{0}^{\Lambda, l a b}>$. Selecting the axial charge related to the particles in the definite rapidity or transverse momentum interval, the respective dependence of polarization may be also obtained.

As the axial charge should be related to the zeroth component of hyperon polarization in laboratory frame $\Pi_{0}^{l a b}$, the transformation to hyperon rest frame should be performed. Taking into account that 


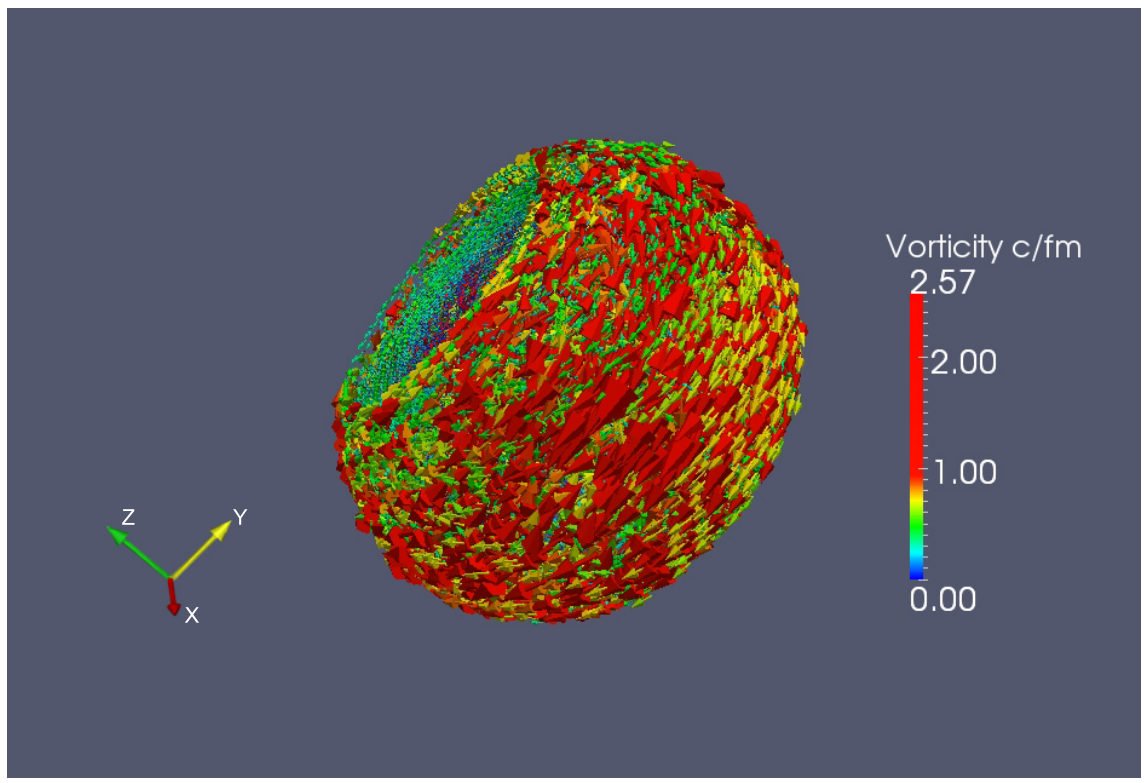

Figure 3. The vortex sheet.

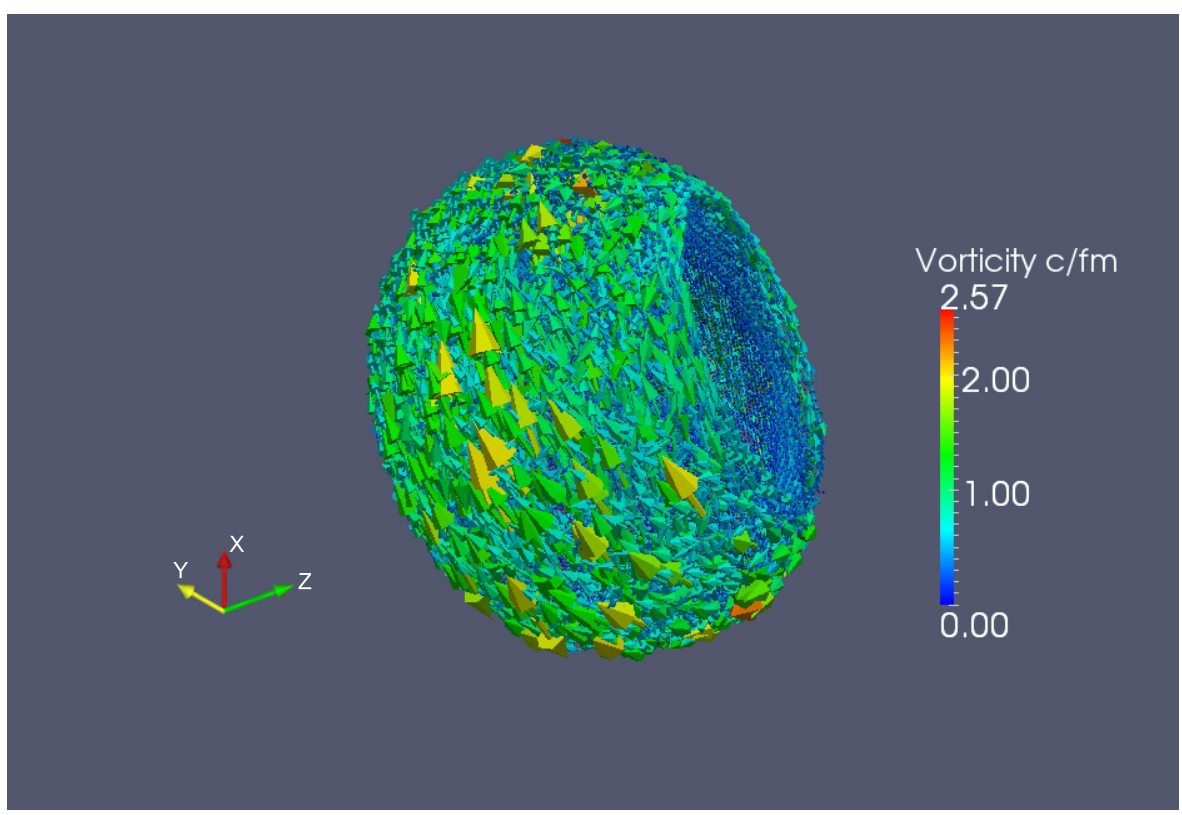

Figure 4. The vortex sheet for the particular direction of angular momentum. 
polarization pseudovector should be directed along $y$ axis (as it has to be collinear to $\vec{L}$ pseudovector), one get

$$
\Pi_{0}^{\Lambda, l a b}=\frac{\Pi_{0}^{\Lambda} p_{y}}{M_{\Lambda}},
$$

so that the rest frame polarization can be obtained as

$$
<\Pi_{0}^{\Lambda}>=Q_{5}^{s}<\frac{M_{\Lambda}}{N_{\Lambda} p_{y}}>.
$$

The possible violation of positivity $\left(\left|\Pi_{\Lambda}\right| \geq 1\right)$ or even the divergence is due to the fact, that hyperons with zero $y$ component of the momentum should not have the zeroth component of polarization and therefore should not contribute to $Q_{5}^{s}$. To avoid this problem one may instead attribute the factor $p_{y} / M$ to each hyperon in the denominator of (4). Nevertheless the comparison (see figure 5) of various approaches shows the similar scale and rapidity dependence of polarization.
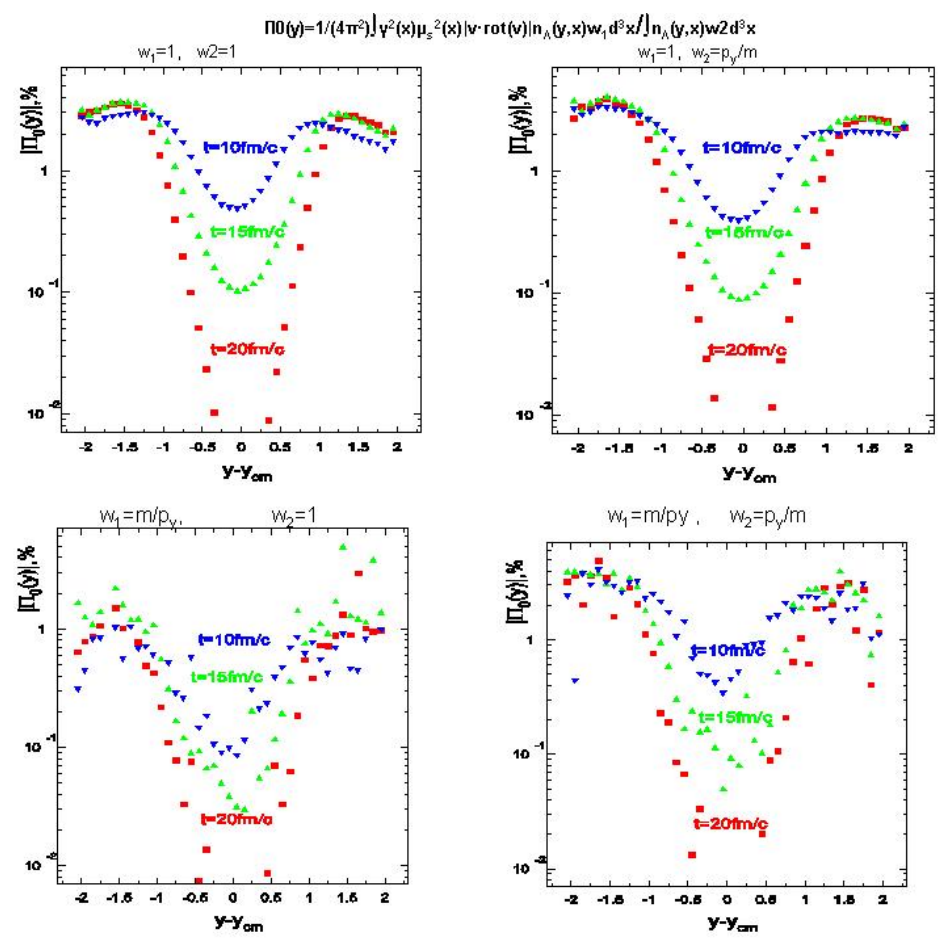

Figure 5. The rapidity dependence of polarization in helicity-based approach.

Another approach to polarization is based on the so-called thermal vorticity [7]. To provide the comparison we calculated (see figure 6) the thermal vorticity field and respective polarization. While a scale of the polarization in the thermal vorticity-based approach is several times larger, its rapidity dependence, surprisingly enough, appear to be similar in these rather distinct approaches.

The current STAR@RHIC measurements of polarization [14] manifested the decrease with energy which is naturally explained in the anomalous mechanism but also happens in the thermal vorticity mechanism. To quantify the predictions, we calculated the energy dependence in the anomalous mechanism, covering the experimental points (see figure 7). 


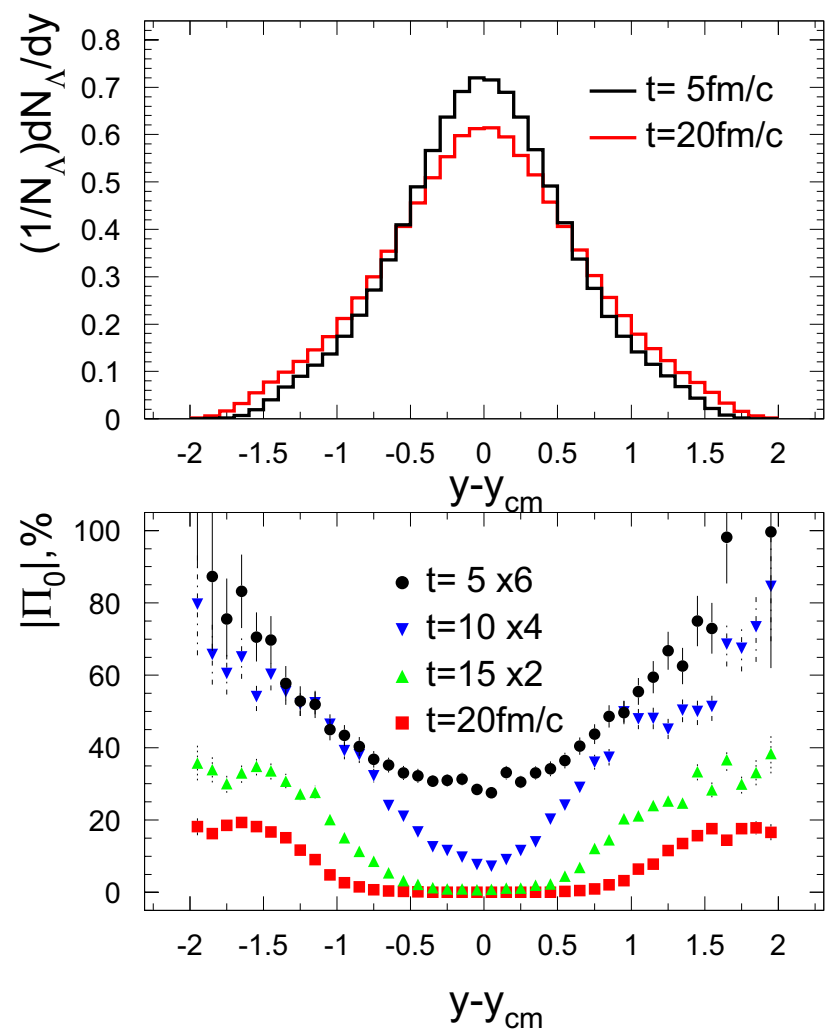

Figure 6. The rapidity dependence of polarization in thermal vorticity-based approach. The magnitudes of polarization at different times are rescaled by the identified factors.

\section{Conclusions and Outlook}

We investigated vorticity and hydrodynamical helicity in noncentral heavy-ion collisions in the framework of the kinetic Quark-Gluon String Model. We have confirmed our earlier observation that the vorticity field is predominantly localized in a relatively thin layer $(2 \div 3 \mathrm{fm})$ on the boundary between the participant and spectator nucleons and observed that it is forming the specific toroidal structures, which might be considered as vortex sheets with the unexpected cylindrical symmetry. They look as cyclones appearing at femtoscopic scale.

The vorticity and helicity fields are manifested in the $\Lambda$ hyperon polarization. We performed its detailed calculations including the simulations of the strange chemical potential. We found that the polarization magnitude might reach a percent level. The comparison with the very different approach 


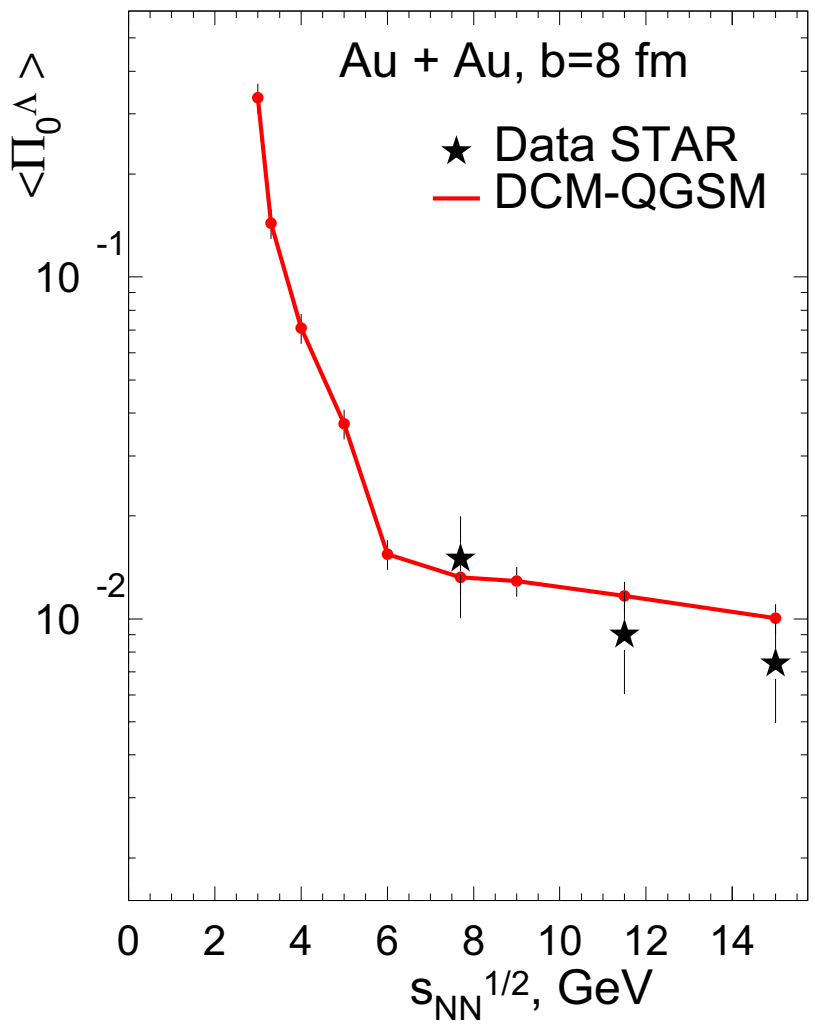

Figure 7. The energy dependence of polarization in anomaly-based approach compared with experimental STAR points.

exploring the thermal vorticity leads to qualitatively similar results, although the polarization scale is several times larger.

\section{Acknowledgements}

This work was supported in part by the Russian Foundation for Basic Research, Grant No. 14-0100647. 


\section{References}

[1] K. Fukushima, D.E. Kharzeev and H.J. Warringa, Phys. Rev. D 78, 074033 (2008). [arXiv:0808.3382 [hep-ph]]

[2] D. Kharzeev and A. Zhitnitsky, Nucl. Phys. A 797, 67 (2007). [arXiv:0706.1026 [hep-ph]]

[3] O. Rogachevsky, A. Sorin and O. Teryaev, Phys. Rev. C 82, 054910 (2010). [arXiv:1006.1331 [hep-ph]]

[4] T. Kalaydzhyan, Phys. Rev. D 89 (10), 105012 (2014). [arXiv:1403.1256 [hep-th]]

[5] M. Baznat, K. Gudima, A. Sorin and O. Teryaev, Phys. Rev. C 88, 061901 (2013). [arXiv:1301.7003 [nucl-th]]

[6] J. -H. Gao, Z. -T. Liang, S. Pu, Q. Wang and X. -N. Wang, Phys. Rev. Lett. 109, 232301 (2012). [arXiv:1203.0725 [hep-ph]]

[7] F. Becattini, L. Csernai and D. J. Wang, Phys. Rev. C 88 (3), 034905 (2013). [arXiv:1304.4427 [nucl-th]]

[8] O. Teryaev and R. Usubov, Phys. Rev. C 92 (1), 014906 (2015)

[9] V. D. Toneev, N. S. Amelin, K. K. Gudima and S. Y. Sivoklokov, Nucl. Phys. A 519, 463C (1990)

[10] N. S. Amelin, E. F. Staubo, L. P. Csernai, V. D. Toneev and K. K. Gudima, Phys. Rev. C 44, 1541 (1991)

[11] J. Aichelin and K. Werner, Phys. Rev. C 79, 064907 (2009). [Phys. Rev. C 81, 029902 (2010)]. [arXiv:0810.4465 [nucl-th]]

[12] J. Steinheimer and M. Bleicher, Phys. Rev. C 84, 024905 (2011). [arXiv:1104.3981 [hep-ph]]

[13] M. I. Baznat, K. K. Gudima, A. S. Sorin and O. V. Teryaev, Phys. Rev. C 93 (3), 031902 (2016). doi:10.1103/PhysRevC.93.031902. [arXiv:1507.04652 [nucl-th]]

[14] M. Lisa [for STAR Collaboration]. Talk at QCD Chirality Workshop, UCLA, February 2016 